\title{
Producción de girasol en función del tipo de suelo, nitrógeno y densidad de población
}

\author{
Sunflower production according to the type of soil, nitrogen, and population density \\ Yasmani Rafael Arenas-Julio ${ }^{1}$ (D) , José Alberto Salvador Escalante-Estrada ${ }^{1}$ (D) , Carlos Alberto \\ Ortiz-Solorio ${ }^{1}$ D , Víctor Hugo Volke-Haller ${ }^{1}$ iD , María Teresa Rodríguez-González ${ }^{1}$ (D), \\ Cid Aguilar-Carpio ${ }^{2 *}$ iD
}

${ }^{1}$ Colegio de Postgraduados, Km 36.5 Carretera México-Texcoco, Montecillo, 56230, Texcoco, Estado de México, México.

${ }^{2}$ IDAGRO S. de R. L. de C. V., Carretera Yautepec-Tlayacapan S/N, Col. Puente Pantitlán, 62545, Tlayacapan, Morelos, México.

*Autor para correspondencia: cid.aguilar.carpio@gmail.com

Fecha de recepción:

4 de enero de 2020

Fecha de aceptación:

25 de enero de 2021

Disponible en línea:

14 de mayo de 2021

Este es un artículo en acceso abierto que se distribuye de acuerdo a los términos de la licencia Creative Commons.

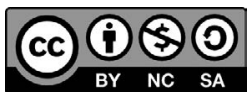

Reconocimiento-

NoComercia-

CompartirIgual 4.0

Internacional

\section{RESUMEN}

El nitrógeno es el nutrimento que limita en mayor medida la producción de girasol (Helianthus annuus L.). El objetivo del estudio fue determinar la producción de biomasa y rendimiento en el cultivo de girasol en función del tipo de suelo, nitrógeno y densidad de población. Los tratamientos fueron tres tipos de suelo donde se aplicaron tres niveles de nitrógeno (0,50 y $\left.100 \mathrm{~kg} \mathrm{ha}^{-1}\right)$, así como tres densidades de población $\left(4,8\right.$ y 12 plantas por $\mathrm{m}^{2}$ ). El diseño fue bloques completos al azar con arreglo de parcelas subdivididas y cuatro repeticiones. Se evaluó la fenología de la planta, biomasa, índice de cosecha, rendimiento y sus componentes. Como resultado se obtuvo que las condiciones climáticas fueron favorables para el desarrollo del cultivo cuando la temperatura osciló entre 8 a $31^{\circ} \mathrm{C}$. De los tres sitios donde se estableció el estudio, el que presentó las mejores condiciones para el crecimiento del cultivo fue en el suelo con pH de 7.1 y CE de $0.49 \mathrm{dS} \mathrm{m}^{-1}$ con la aplicación de 100 $\mathrm{kg} \mathrm{ha}^{-1}$ de nitrógeno y una densidad de 12 plantas $\mathrm{m}^{-2}$. Por ello, la mejor producción de biomasa y rendimiento se encontró en el suelo con $\mathrm{pH}$ neutro, sin salinidad con textura franco arenoso en combinación con el más alto nivel de nitrógeno y densidad de población.

\section{PALABRAS CLAVE}

Biomasa, rendimiento de grano, fertilización.

\section{ABSTRACT}

Nitrogen is the nutrient that most limits sunflower (Helianthus annuus L.) production. The objective of this study was to determine the influence of soil type, nitrogen and population density on biomass production and grain yield of sunflower crop. The treatments consisted of three soil types with the application of three levels of nitrogen $\left(0,50\right.$ and $\left.100 \mathrm{~kg} \mathrm{ha}^{-1}\right)$, as well as three population densities $\left(4,8\right.$ and 12 plants $\left.\mathrm{m}^{-2}\right)$. The design included complete blocks at random with subdivided plots and four replications. We evaluated the plants phenology, biomass, harvest index, grain yield and its components. The results showed that the climatic conditions were favorable for the development of the crop with temperatures between 8 to $31^{\circ} \mathrm{C}$. Of the three sites studied, the one that presented the best conditions for crop growth was the soil with a $\mathrm{pH}$ of 7.1 and an $\mathrm{EC}$ of 0.49 $\mathrm{dS} \mathrm{m} \mathrm{m}^{-1}$ with the application of $100 \mathrm{~kg} \mathrm{ha}^{-1}$ of nitrogen and a density of 12 plants $\mathrm{m}^{-2}$. Therefore, the best biomass production and grain yield was achieved in soil with neutral $\mathrm{pH}$, without salinity with sandy franc texture in combination with the highest nitrogen level and population density.

\section{KEYWORDS}

Biomass, grain yield, fertilization. 


\section{INTRODUCCIÓN}

El girasol (Helianthus annuus L.) es uno de los cultivos más importante en la producción de aceite, por su alto contenido de grasas poliinsaturadas (García y Quinche 2012), bien adaptado a diversas condiciones de salinidad del suelo (13.8 $\left.\mathrm{dS} \mathrm{m}^{-1}\right)$, ya que no afecta la producción de grano y puede ser más rentable que cultivos como el maíz y el frijol en esta condición de suelo (Escalante-Estrada y Rodríguez-González 2010; Escalante-Estrada et al. 2015). México importa aproximadamente $85 \%$ de semillas oleaginosas para la extracción de aceite comestible. Para 2018, la producción nacional de girasol fue de 9,263 t, en una superficie cosechada de $6,629 \mathrm{ha}^{-1}$, con un rendimiento promedio de $1.39 \mathrm{t} \mathrm{ha}^{-1}$; los principales estados productores fueron Sonora, Zacatecas, Guanajuato, Jalisco y el Estado de México (SIAP 2018).

Desde el punto de vista agronómico, la tolerancia a la salinidad se define como la habilidad de las plantas de sobrevivir y producir rendimientos económicos en condiciones de estrés y se expresa como la relación entre el rendimiento de una variedad en condiciones salinas respecto a su rendimiento en condiciones normales (González et al. 2002). Investigaciones efectuadas con girasol en condiciones de salinidad indican un efecto negativo en el desarrollo de la planta y rendimiento del cultivo (Habeen y Ahmad 2012). En las áreas dedicadas a la agricultura, la obtención de rendimientos altos cada vez está más restringida debido a la salinidad de los suelos. Se estima que 800 millones de hectáreas en el planeta están afectadas por sales; de éstas, 397 millones son por problemas de salinidad y 434 millones por la sodicidad del suelo (Munns et al. 2005). Por ello, es necesario generar información sobre el manejo del cultivo en diferentes condiciones ambientales, las cuales promuevan su siembra y producción, mediante el uso de prácticas agrícolas (fertilización y topografía) que provean a los cultivos las condiciones más favorables para la expresión de un mayor rendimiento.

En el caso del girasol, el déficit de nitrógeno es lo que limita su producción en mayor medida, debido a que es importante en la estructura de proteínas y ácidos nucleicos, y, en conjunto con el carbono, podría considerarse como uno de los elementos centrales para la producción de este cultivo (García y Quinche 2012; Escalante-Estrada y Rodríguez-González
2010). Por otro lado, el incremento en la densidad de población genera mayor sombreado y afecta procesos fisiológicos (fotosíntesis, respiración, fotorrespiración y transpiración), así como un incremento en la acumulación de biomasa, lo que se manifiesta en el índice de cosecha y rendimiento de grano (Escalante-Estrada et al. 2020). Asimismo, se han reportado trabajos efectuados en girasol en los cuales se indica que la densidad de población y fertilización nitrogenada son prácticas esenciales para obtener una mayor producción (Ali y Noorka 2013; Rasool et al. 2013; Arenas-Julio et al. 2021). Por lo anterior, el objetivo de este estudio fue determinar la producción de biomasa y rendimiento de grano en el cultivo de girasol en función del tipo de suelo, nitrógeno y densidad de población.

\section{Materiales y Métodos}

El estudio se desarrolló en el campus experimental del Colegio de Postgraduados, Montecillos, Estado de México, México (19²7’ 48.74" N, 98 54' 22.34” O, 2250 $\mathrm{m}$ de altitud); con clima BS1, que corresponde al menos seco de los áridos, con régimen de lluvias en verano, temperatura media anual de $14.6^{\circ} \mathrm{C}$ y precipitación media anual de $500 \mathrm{~mm}$ (García 2004). Como material vegetativo se empleó la variedad de girasol Victoria, el cual se sembró en tres diferentes suelos (sitios). Previo al establecimiento del estudio, los suelos fueron analizados para determinar sus propiedades fisicoquímicas (Cuadro 1). En cada sitio se aplicaron tres niveles de nitrógeno (N) 0, 50 y $100 \mathrm{~kg} \mathrm{ha}^{-1}$, en combinación con tres densidades de población de 4, 8 y 12 plantas $\mathrm{m}^{-2}$ (DP4, DP8 y DP12, respectivamente), lo que originó un total de 27 tratamientos.

El diseño experimental fue de bloques completos al azar, con arreglo de parcelas sub-subdivididas en cuatro repeticiones. La parcela mayor correspondió al tipo de suelo, la mediana a los niveles de nitrógeno y la menor a las densidades de población.

Durante el desarrollo del estudio, se registraron la temperatura (máxima y mínima diaria), así como la evaporación y la precipitación, las cuales fueron obtenidas de la estación agrometeorológica del Colegio de Postgraduados. En lo que respecta al cultivo, se registró la ocurrencia de las etapas fenológicas [días a emergencia (E), floración (R5) 
Cuadro 1. Características de los suelos donde se estableció el estudio.

\begin{tabular}{|c|c|c|c|c|}
\hline Sitio & Textura del suelo & $\mathrm{pH}$ & $\begin{array}{l}\text { Conductividad eléctrica } \\
\qquad\left(\mathrm{dS} \mathrm{m}^{-1}\right)\end{array}$ & $\begin{array}{c}\text { Ubicación } \\
(2,244 \mathrm{msnm})\end{array}$ \\
\hline Suelo 1 & Franco arenoso & 7.1 & 0.49 & $\begin{array}{l}19^{\circ} 27^{\prime} 41.65^{\prime \prime} \mathrm{N} \\
98^{\circ} 54^{\prime} 22.02^{\prime \prime} \mathrm{O}\end{array}$ \\
\hline Suelo 2 & Franco arcilloso limoso & 7.8 & 1.75 & $\begin{array}{l}19^{\circ} 28^{\prime} 7.88^{\prime \prime} \mathrm{N} \\
98^{\circ} 54^{\prime} 12.02 ” \mathrm{O}\end{array}$ \\
\hline Suelo 3 & Arcillosa & 8.1 & 5.11 & $\begin{array}{l}19^{\circ} 27^{\prime} 57.97 ” \mathrm{~N} \\
98^{\circ} 54 ' 43.53 ” \mathrm{O}\end{array}$ \\
\hline
\end{tabular}

y madurez fisiológica (R9)] (Schneiter y Miller 1981). En cada etapa, se determinó la acumulación de unidades calor (UC), mediante el método residual descrito por Flores et al. (1985):

Dónde: Tmáx = Temperatura máxima diaria $\left({ }^{\circ} \mathrm{C}\right)$, Tmín $=$ Temperatura mínima diaria $\left({ }^{\circ} \mathrm{C}\right)$ y $\mathrm{TB}=$ Temperatura base, considerada como $8^{\circ} \mathrm{C}$ (Sadras y Hall 1988). La evapotranspiración del cultivo (ETc) fue estimada a partir de los datos de evaporación (Ev), obtenidos de un tanque tipo " $\mathrm{A}$ ", en la que se emplearon los coeficientes 0.6 y 0.8 , correspondientes al dispositivo y cultivo, respectivamente (Allen et al. 2006).

$$
U C=\left(\frac{\text { Tmáx }+ \text { Tmín }}{2}\right)-T B
$$

En la cosecha se registró el rendimiento de grano $\left(\mathrm{RG}, \mathrm{g} \mathrm{m}^{-2}\right.$ ) y sus componentes área del capítulo (AC) y peso de 100 semillas (P100S, g); también se obtuvo la biomasa total (BT, g) (peso de la materia seca + rendimiento de grano) y se calculó el índice de cosecha (IC\% = Rendimiento de grano/biomasa total). A los datos obtenidos se les efectuó un análisis de varianza y prueba de comparación múltiple de medias, mediante la prueba de Tukey $(\mathrm{P} \leq 0.05)$, en la que se empleó el paquete estadístico SAS versión 9.1.

\section{RESUlTAdos Y Discusión}

\section{Elementos del clima y fenología}

En la Figura 1, se presentan los datos de temperatura máxima (Tmáx), mínima (Tmín), promedio decenal y la precipitación suma decenal, durante el ciclo del cultivo; se observa que la Tmáx fluctuó entre 31 y $21^{\circ} \mathrm{C}$ y la Tmín de 13 a $8^{\circ} \mathrm{C}$. Dichos valores térmicos son apropiados para el desarrollo del cultivo de girasol (Escalante-Estrada y Rodríguez-González 2010; Escalante-Estrada et al. 2015). La temperatura más alta $\left(31^{\circ} \mathrm{C}\right)$ se dio en junio y disminuyó a medida que se incrementó la precipitación, al momento de la emergencia del cultivo. $\mathrm{Al}$ respecto, Arenas-Julio et al. (2021) reportaron tendencias similares al evaluar la fenología de girasol en condiciones de temporal, en Montecillo, Estado de México. La lluvia estacional acumulada fue de $388 \mathrm{~mm}$, de la cual $69 \%$ ocurrió desde la E a R5 (270 mm), lo cual indica que el cultivo no presentó deficiencia hídrica en la etapa vegetativa. En la época de floración y llenado de grano, incidieron $118 \mathrm{~mm}$, que representan 31\% de la precipitación durante el ciclo del cultivo. Esto pudo limitar una mayor expresión del rendimiento de grano.

En los suelos evaluados, las etapas fenológicas ocurrieron en tiempo similar; no se observaron cambios en la fenología debido al suelo, nitrógeno y densidad de población. Lo anterior, posiblemente se debió a que la nutrición en los diferentes tipos de suelo fue suficiente para que la planta lograra expresar al mismo tiempo sus etapas fenológicas. Así, la E de plántulas de girasol se presentó a los 7 días después de la siembra (DDS), la floración ocurrió a los 65 DDS y la etapa R9 se presentó a los 130 DDS (Figura 1). Estos resultados concuerdan parcialmente con los reportados por Escalante-Estrada y Rodríguez-González (2010), al evaluar la fenología de girasol en tres niveles de salinidad del suelo y en agrosistemas (girasol-frijol), en donde la E se presentó a los 8 DDS, el periodo de R5 ocurrió a los 80 DDS y R9 a los 130 DDS. 


\section{Unidades calor y evapotranspiración}

La acumulación de unidades calor (UC) y evapotranspiración acumulada del cultivo (ETc), en relación con la fenología durante el ciclo del cultivo, mostró una relación lineal con el tiempo que respondió al modelo $\mathrm{y}=\mathrm{a}+\mathrm{bx}$. Así, las UC y ETc fueron $144^{\circ} \mathrm{C} \mathrm{d}^{-1}$ y $39 \mathrm{~mm}$ a la emergencia, $866^{\circ} \mathrm{C} \mathrm{d}^{-1}$ y $121 \mathrm{~mm}$ a la floración y $1,510^{\circ} \mathrm{C} \mathrm{d}^{-1}$ y $122 \mathrm{~mm}$ a la madurez fisiológica. La ETc acumulada durante todo el ciclo del cultivo fue de $282 \mathrm{~mm}$ (Figura 2).

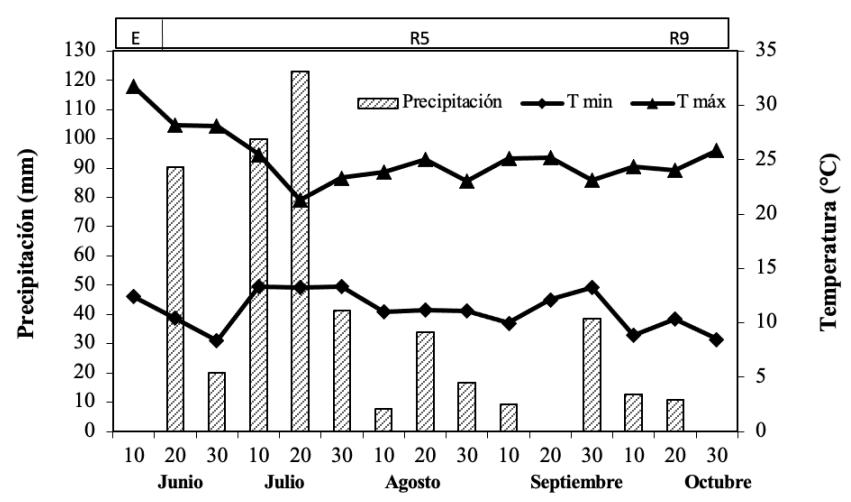

Figura 1. Temperatura máxima, mínima (media decenal) y precipitación (suma decenal) durante el ciclo de cultivo de girasol. $\mathrm{E}=$ Emergencia, R5 = Floración y R9 = Madurez fisiológica. Montecillo, Estado de México, México.

Lo anterior indica que las necesidades de agua del cultivo fueron semejantes entre las diferentes localidades en estudio, pues esto está relacionado con el ciclo del cultivo. Escalante-Estrada et al. (2015) encontraron que el cv. Victoria en clima templado (Montecillo, México) requirió 134,734 y $1004 \mathrm{UC}\left({ }^{\circ} \mathrm{C}\right.$ $\left.\mathrm{d}^{-1}\right)$ a la emergencia, floración y madurez fisiológica, respectivamente, datos similares a los encontrados en el presente estudio. Por su lado, Escalante-Estrada y Rodríguez-González (2010) reportaron en girasol para el cv. Victoria (Montecillo, México) una evaporación total de $589 \mathrm{~mm}$, datos que pueden diferir debido a la época en la que se llevó a cabo el estudio.

En el Cuadro 2, se presenta el análisis de varianza de los factores de estudio y sus interacciones, el cual muestra que para BT, IC y AC existen diferencias significativas en $S, N, D P, S^{*} N, S^{*} D P, N^{*} D P$ y $S^{*} N^{*} D P$; mientras que para RG la interacción que no ocasionó cambios significativos fue $\mathrm{N}^{*} \mathrm{DP}$ y en P100S la no significancia fue en $N, S^{*} D P$ y $N^{*} D P$.

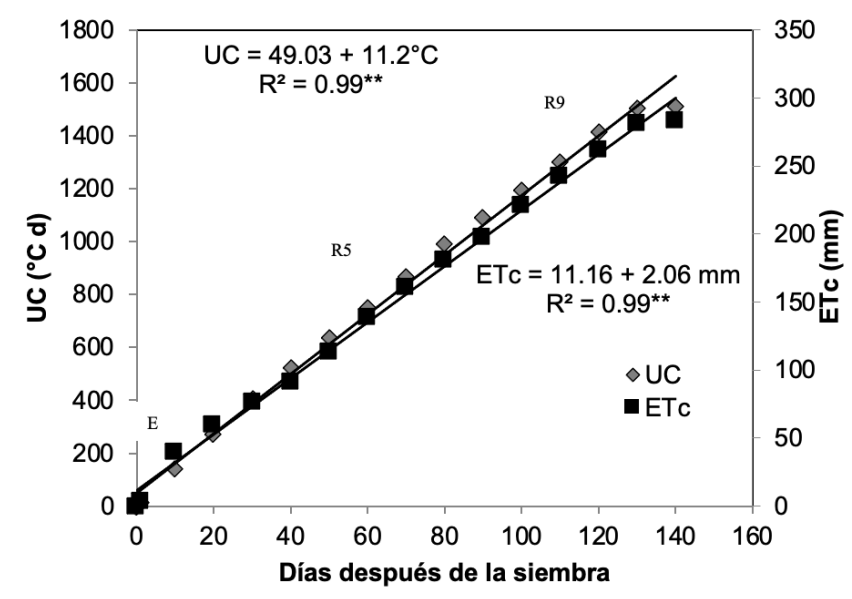

Figura 2. Unidades calor (UC, ${ }^{\circ} \mathrm{C} \mathrm{d}^{-1}$ ) y Evapotranspiración (ETc, mm) acumuladas en el cultivo de girasol cv. Victoria. $\mathrm{E}=$ Emergencia, R5 = Floración, R9 = Madurez fisiológica.

\section{Tipo de suelo (S)}

La producción de BT, IC, RG, P100S y AC más alta se observó en S1, el cual superó en 42.5, 4, 25.5, 12.5 y $36.2 \%$ en comparación a S2. Cabe señalar que, en S3 fue donde se registró la producción más baja, en relación con S1 y S2 (Cuadro 2). Al respecto, Dos-Santos et al. (2017) señalan que la reducción en la producción de las plantas en estrés salino se explica por exceso de acumulación de sales $(\mathrm{NaCl})$ en los tejidos vegetales, disminución en el potencial osmótico de la solución del suelo, posible toxicidad iónica e inestabilidad nutricional. Por otro lado, Escalante-Estrada y Rodríguez-González (2010), al evaluar girasol en suelos con problemas de salinidad (CE 5, 7 y $11 \mathrm{dS}^{-1}$ ), encontraron que el suelo con la menor salinidad (CE $5 \mathrm{dS} \mathrm{m}^{-1}$ ) fue el de mayor producción de biomasa (1055 $\left.\mathrm{g} \mathrm{m}^{-2}\right)$ y RG $\left(102 \mathrm{~g} \mathrm{~m}^{-2}\right)$, tendencias que se relacionan con el presente estudio.

\section{Niveles de nitrógeno $(\mathrm{N})$}

Con la aplicación de $100 \mathrm{~kg} \mathrm{ha}^{-1}$ de nitrógeno, la producción de BT, RG y el AC aumentaron en 23, 13 y 21\%, respectivamente, en comparación con la aplicación de 50 y $0 \mathrm{~kg} \mathrm{ha}^{-1}$ de nitrógeno. No obstante, estadísticamente no existieron diferencias entre la aplicación de 50 y $100 \mathrm{~kg} \mathrm{ha}^{-1}$ de nitrógeno en el RG, IC y P100S, pero sí con respecto a la nula fertilización. Esto confirma que el nitrógeno es 
Cuadro 2. Análisis de varianza para la biomasa total (BT), índice de cosecha (IC), rendimiento de grano (RG), peso de 100 semillas (P100S) y área de capítulo (AC), en función al tipo de suelo (S), niveles de nitrógeno (N) y densidad de población (DP).

\begin{tabular}{cccccc}
\hline \multirow{2}{*}{ FACTOR } & BT & IC & RG & P100S & AC \\
& $\left(\mathrm{g} \mathrm{m}^{-2}\right)$ & $(\%)$ & $\left(\mathrm{g} \mathrm{m}^{-2}\right)$ & $(\mathrm{g})$ & $* *$ \\
\hline $\mathrm{S}$ & $* *$ & $* *$ & $* *$ & $\mathrm{~cm})^{2}$ & $* *$ \\
$\mathrm{~N}$ & $* *$ & $* *$ & $* *$ & $* *$ & $* *$ \\
$\mathrm{DP}$ & $* *$ & $* *$ & $* *$ & $*$ & $* *$ \\
$\mathrm{~S} * \mathrm{DP}$ & $* *$ & $* *$ & $* *$ & $\mathrm{NS}$ & $*$ \\
$\mathrm{~N} * \mathrm{DP}$ & $* *$ & $* *$ & $\mathrm{NS}$ & $*$ \\
$\mathrm{~S} * \mathrm{~N} * \mathrm{DP}$ & $* *$ & $* *$ & $* *$ & $* *$ & $* *$ \\
\hline
\end{tabular}

$*=\mathrm{P} \leq 0.05, * *=\mathrm{P} \leq 0.01, \mathrm{NS}=$ Diferencias no significativas a $\mathrm{P} \geq 0.05$.

esencial para el desarrollo del cultivo de girasol, por incrementar la BT, IC, RG, AC y P100S (Morales et al. 2015; Hawkesford et al. 2012). Escalante-Estrada et al. (2020), al evaluar en girasol diferentes niveles de nitrógeno, encontraron un incremento significativo en la BT y RG. Al respecto, Ali y Noorka (2013), con 85 a $185 \mathrm{~kg} \mathrm{ha}^{-1}$ de nitrógeno lograron incrementar en $28 \%$ la producción de grano en girasol.

\section{Densidad de población (DP)}

Durante el desarrollo del cultivo, la producción de BT y RG por $\mathrm{m}^{2}$ mostró un incremento significativo por el aumento en la densidad de población (Cuadro 3). Así, la BT y RG más baja se presentó en la DP4, seguido de la DP8 donde la producción se incrementó en 92 (BT) y 51 (RG) \%; en DP12 (12 plantas $\left.\mathrm{m}^{-2}\right)$, el aumento fue de $160(\mathrm{BT})$ y 51 (RG) \%, la producción más alta en comparación con DP4 (Cuadro 2). Lo anterior indica que con la mayor densidad de plantas se logra un mayor dosel vegetal, lo que representa una mayor captación de radiación interceptada. Asimismo, aumentos en la radiación interceptada por el cultivo incrementan la producción de fotoasimilados, lo que se refleja en un aumento en el rendimiento de grano (Morales-Rosales et al. 2008). Por otro lado, el IC, P100S y AC más bajo se observó en la DP12 en comparación a DP4. Al aumentar la DP, se observó una reducción en los componentes del rendimiento; tendencias similares observaron Escalante-Estrada et al. (2020) y Escalante-Estrada et al. (2008), quienes indican que los órganos de la planta son afectados por la competencia de luz, agua y nutrimentos al incrementar la densidad de siembra.

\section{Interacción suelo $\mathrm{x}$ nitrógeno}

En general, los tres tipos de suelo presentaron los valores de BT, IC, RG, P100S y AC más alta con la aplicación de $100 \mathrm{~kg} \mathrm{ha}^{-1}$ de nitrógeno, en comparación con 50 y $0 \mathrm{~kg} \mathrm{ha}^{-1}$ de nitrógeno; en esta última se obtuvieron los valores más bajos. En el caso del IC, RG y P100S, no se observaron diferencias entre la aplicación 100 y $50 \mathrm{~kg} \mathrm{ha}^{-1}$ de nitrógeno (Cuadro 4). $\mathrm{Al}$ respecto, Rasool et al. (2013), al evaluar diferentes niveles de nitrógeno, lograron aumentar la BT y RG, tendencias similares al presente estudio. De los tres tipos de suelo evaluados, el que presentó la mayor producción de BT y RG fue S1 (pH de 7.1 y CE de 0.49 $\mathrm{dS} \mathrm{m}^{-1}$ ) con la aplicación de N100, cuyo incremento fue de 55 y $42 \%$ respecto a N0 y S3 (pH de 8.1 y CE de $5.11 \mathrm{dS} \mathrm{m}^{-1}$ ), el cual registró las menores $\mathrm{BT}, \mathrm{RG}$, P100S y AC. Lo anterior se debió a que los niveles altos de salinidad disminuyen los parámetros de crecimiento y limitan la producción agrícola, debido a la disminución de la actividad fotosintética, así como a modificaciones morfológicas, estructurales y metabólicas en las plantas (Conus et al. 2009; Dos-Santos et al. 2017).

\section{Interacción suelo $\mathrm{x}$ densidad de población}

Para la interacción S*DP (Figura 3), la mayor producción de biomasa y rendimiento de grano 
Cuadro 3. Biomasa total (BT), índice de cosecha (IC), rendimiento de grano (RG), peso de 100 semillas (P100S) y área de capítulo $(\mathrm{AC})$ de girasol en función del tipo suelo (S), niveles de nitrógeno (N) y densidad de población (DP). Factores principales.

\begin{tabular}{cccccc}
\hline FACTOR & $\begin{array}{c}\text { BT } \\
\left(\mathrm{g} \mathrm{m}^{-2}\right)\end{array}$ & $\begin{array}{c}\text { IC } \\
(\%)\end{array}$ & $\begin{array}{c}\text { RG } \\
\left(\mathrm{g} \mathrm{m}^{-2}\right)\end{array}$ & $\begin{array}{c}\text { P100S } \\
(\mathrm{g})\end{array}$ & $\begin{array}{c}\text { AC } \\
\left(\mathrm{cm}^{2}\right)\end{array}$ \\
\hline S1 & $1365 \mathrm{a}^{\mathrm{II}}$ & $30 \mathrm{a}$ & $346 \mathrm{a}$ & $8 \mathrm{a}$ & $315 \mathrm{a}$ \\
S2 & $784 \mathrm{~b}$ & $29 \mathrm{~b}$ & $258 \mathrm{~b}$ & $7 \mathrm{~b}$ & $201 \mathrm{~b}$ \\
S3 & $777 \mathrm{~b}$ & $25 \mathrm{c}$ & $228 \mathrm{c}$ & $6 \mathrm{c}$ & $95 \mathrm{c}$ \\
DMS & 47.5 & 1.0 & 6.1 & 0.6 & 19.9 \\
N0 & $871 \mathrm{c}$ & $27 \mathrm{~b}$ & $255 \mathrm{~b}$ & $7 \mathrm{a}$ & $189 \mathrm{~b}$ \\
N50 & $981 \mathrm{~b}$ & $28 \mathrm{ab}$ & $288 \mathrm{a}$ & $7 \mathrm{a}$ & $191 \mathrm{~b}$ \\
N100 & $1075 \mathrm{a}$ & $29 \mathrm{a}$ & $288 \mathrm{a}$ & $7 \mathrm{a}$ & $230 \mathrm{a}$ \\
DMS & 43.3 & 1.2 & 6.4 & 0.4 & 17.2 \\
DP4 & $524 \mathrm{c}$ & $31 \mathrm{a}$ & $191 \mathrm{c}$ & $7 \mathrm{a}$ & $238 \mathrm{a}$ \\
DP8 & $1010 \mathrm{~b}$ & $28 \mathrm{~b}$ & $289 \mathrm{~b}$ & $7 \mathrm{a}$ & $200 \mathrm{~b}$ \\
DP12 & $1392 \mathrm{a}$ & $25 \mathrm{c}$ & $350 \mathrm{a}$ & $6 \mathrm{~b}$ & $172 \mathrm{c}$ \\
DMS & 28.7 & 1.5 & 3.8 & 0.2 & 16.9 \\
CV & 3.4 & 2.3 & 2.5 & 3.9 & 6.1 \\
\hline
\end{tabular}

${ }^{\mathbb{L}}$ Letras distintas en la misma columna indican diferencias significativas, según Tukey $=0.05$. Suelo 1 (S1), pH de 7.1 y CE de 0.49 dS m ${ }^{-1}$, suelo 2 (S2), pH de $7.8, \mathrm{CE}$ de $1.75 \mathrm{dS} \mathrm{m}^{-1}$ y Suelo 3 (S3), pH de 8.1 y CE de $5.11 \mathrm{dS} \mathrm{m}^{-1}$; N0, N50 y N100 = 0, 50 y $100 \mathrm{~kg} \mathrm{ha}^{-1}$ de nitrógeno. DP4, DP8 y DP12 = 4, 8 y 12 plantas m².

se generó en S1 y DP12, con un incremento de 78 y $65 \%$ respecto a DP4. Elevar el número de plantas por superficie genera un aumento en fotosíntesis, debido al crecimiento del dosel vegetal, lo que, a su vez, repercute en una mayor producción (Escalante-Estrada y Rodríguez-González 2010). Cabe señalar que S1 presenta un suelo con $\mathrm{pH}$ neutro $(\mathrm{pH}$ de 7.1) y CE de $0.49 \mathrm{dS} \mathrm{m}^{-1}$, condiciones posiblemente óptimas para el desarrollo de la planta, pues el aumento en la salinidad produce una reducción en la BT y RG, como lo observaron Escalante-Estrada y Rodríguez-González (2010), quienes registraron una reducción en la BT a medida que aumentaba la CE. Las tendencias de la BT y RG en función de la densidad de población

Cuadro 4. Biomasa total (BT), índice de cosecha (IC), rendimiento de grano (RG) y sus componentes en el cultivo de girasol cv. Victoria en función del suelo y niveles de nitrógeno.

\begin{tabular}{|c|c|c|c|c|c|c|}
\hline SUELO & $\begin{array}{c}\mathrm{N} \\
\left(\mathrm{kg} \mathrm{ha}^{-1}\right)\end{array}$ & $\begin{array}{c}\mathrm{BT} \\
\left(\mathrm{g} \mathrm{m}^{-2}\right)\end{array}$ & $\begin{array}{l}\text { IC } \\
(\%)\end{array}$ & $\begin{array}{c}\mathrm{RG} \\
\left(\mathrm{g} \mathrm{m}^{-2}\right)\end{array}$ & $\begin{array}{l}\text { P100S } \\
\text { (g) }\end{array}$ & $\begin{array}{c}\mathrm{AC} \\
\left(\mathrm{cm}^{2}\right)\end{array}$ \\
\hline & 0 & $1221 c^{\mathbb{I}}$ & $23 \mathrm{e}$ & $332 \mathrm{~b}$ & 7 a & $289 \mathrm{~b}$ \\
\hline \multirow[t]{3}{*}{$\mathrm{S} 1$} & 50 & $1337 \mathrm{~b}$ & $27 c$ & 351 a & $8 a$ & $290 \mathrm{~b}$ \\
\hline & 100 & 1537 a & $25 \mathrm{~d}$ & 353 a & $8 a$ & $365 \mathrm{a}$ \\
\hline & 0 & $697 \mathrm{e}$ & $29 \mathrm{~b}$ & $225 \mathrm{e}$ & $6 \mathrm{~b}$ & $190 \mathrm{c}$ \\
\hline \multirow[t]{3}{*}{ S2 } & 50 & $791 \mathrm{~d}$ & $31 \mathrm{a}$ & $267 \mathrm{c}$ & 7 a & $193 \mathrm{c}$ \\
\hline & 100 & $865 d$ & $31 \mathrm{a}$ & $281 \mathrm{c}$ & $7 \mathrm{ab}$ & $218 c$ \\
\hline & 0 & $694 \mathrm{e}$ & $29 \mathrm{~b}$ & $207 \mathrm{f}$ & $6 \mathrm{~b}$ & $84 \mathrm{~d}$ \\
\hline \multirow[t]{2}{*}{ S3 } & 50 & $815 \mathrm{~d}$ & $29 \mathrm{~b}$ & 232 de & $6 \mathrm{~b}$ & $93 \mathrm{~d}$ \\
\hline & 100 & $823 \mathrm{~d}$ & $29 \mathrm{~b}$ & $244 \mathrm{~d}$ & $6 \mathrm{~b}$ & $106 \mathrm{~d}$ \\
\hline DMS & & 83.3 & 1.7 & 13.2 & 0.8 & 32.3 \\
\hline $\mathrm{CV}$ & & 3.5 & 2.5 & 1.9 & 4.7 & 6.6 \\
\hline
\end{tabular}

`Letras distintas en la misma columna indican diferencias significativas, según Tukey = 0.05. P100S = Peso de 100 semillas; AC = Área del capítulo. Suelo 1 (S1), pH de 7.1 y CE de $0.49 \mathrm{dS} \mathrm{m}^{-1}$, suelo 2 (S2), pH de 7.8, CE de $1.75 \mathrm{dS} \mathrm{m}^{-1}$ y Suelo 3 (S3), pH de 8.1 y CE de $5.11 \mathrm{dS} \mathrm{m}^{-1}$. 
para los tres tipos de suelo se ajustaron a un modelo cuadrático. Así, la mayor producción de BT y RG por densidad de planta sembrada se presentó en S1 (23.8 y $4.1 \mathrm{~g} \mathrm{~m}^{-2}$ ), en comparación con S2 y S3. Esto indica que la reducción del crecimiento de las plantas en estrés salino esta relacionada con el exceso de acumulación de ciertos iones en los tejidos vegetales, causado por una posible toxicidad iónica e inestabilidad nutricional en el suelo (Dos-Santos et al. 2017). Silva et al. (2009) verificaron que el aumento de la conductividad eléctrica en el suelo redujo significativamente la acumulación de biomasa total en girasol ornamental.

\section{Interacción nitrógeno $\mathrm{x}$ densidad de población}

En la interacción $\mathrm{N}^{*} \mathrm{DP}$, la densidad a 12 plantas $\mathrm{m}^{-2}$ y $100 \mathrm{~kg} \mathrm{ha}^{-1}$ de nitrógeno obtuvo el mayor RG (364 g $\mathrm{m}^{-2}$ ) y BT $\left(1548 \mathrm{~g} \mathrm{~m}^{-2}\right)$, en comparación con DP8 y DP12 (Figura 4). La aplicación de $100 \mathrm{~kg} \mathrm{~N} \mathrm{ha-2} \mathrm{favoreció}$ la translocación de fotoasimilados en la planta, al incrementar el RG y BT en las tres DP. Por otro lado, Süzer (2010), al aplicar 0, 60, $120 \mathrm{~kg} \mathrm{ha}^{-1}$ de nitrógeno y con densidad de población de 14, 9 y 7 plantas $\mathrm{m}^{-2}$, obtuvo un RG de $258 \mathrm{~g} \mathrm{~m}^{-2}$ con la fertilización más alta. Las tendencias de la BT y RG en función de los niveles se observó en DP12 (2.53 y $1.02 \mathrm{~g} \mathrm{~m}^{-2}$ ), en comparación con DP8 y DP4. Esto indica que el suministro de nitrógeno es necesario para aumentar la acumulación de biomasa en el tallo y órganos de la planta; es decir, al incrementar la densidad de población se genera un mayor dosel vegetal, lo que potencializa la producción del cultivo (BT y RG), al tener una mayor radiación interceptada (Escalante-Estrada et al. 2020). Al respecto, Escalante-Estrada et al. (2020) observaron en el cultivo de girasol que con la aplicación de $100 \mathrm{~kg} \mathrm{ha}^{-1}$ de nitrógeno y densidad de 100,000 plantas ha-1 se logró incrementar el rendimiento y biomasa total (219 y $1220 \mathrm{~g} \mathrm{~m}^{-2}$ ), en comparación con la densidad de 50,000 plantas ha- ${ }^{-1}$.

\section{Interacción suelo $\mathrm{x}$ nitrógeno $\mathrm{x}$ densidad de población}

Para la interacción $\mathrm{S}^{*} \mathrm{~N}^{*} \mathrm{DP}$ (Cuadro 5), la BT y el RG más alto (2173 y $437 \mathrm{~g} \mathrm{~m}^{-2}$, respectivamente) se obtuvieron con la combinación de S1, N100 y DP12, que fue superior en 42 y $12 \%$ respecto a $S 2$ y en 43 y $33 \%$ en comparación con S3. La baja salinidad (CE de $0.49 \mathrm{dS} \mathrm{m}^{-1}$ ) y el pH neutro (7.1) presentados en S1 favorecieron la producción del cultivo en comparación con S2 y S3, donde la CE fue más alta de $1.75 \mathrm{dS} \mathrm{m}^{-1}$

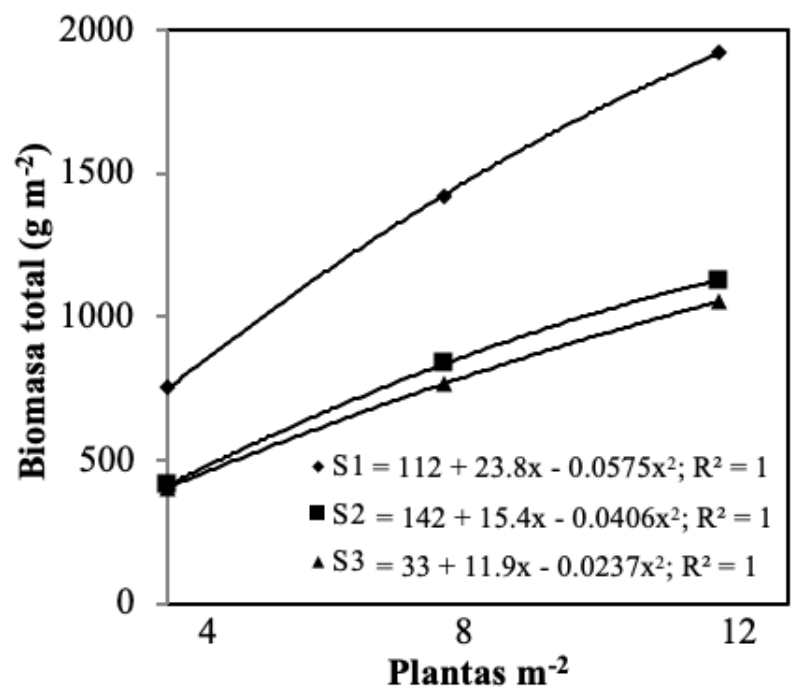

Figura 3. Biomasa total y rendimiento de grano del girasol en función a la densidad de población $\left(4,8\right.$ y 12 plantas $\left.\mathrm{m}^{-2}\right)$ y suelo $\left(\mathrm{S} 1, \mathrm{pH}\right.$ de 7.1 y CE de $0.49 \mathrm{dS} \mathrm{m}^{-1}$, S2, $\mathrm{pH}$ de $7.8, \mathrm{CE}$ de $1.75 \mathrm{dS} \mathrm{m}^{-1}$ y S3, $\mathrm{pH}$ de 8.1 y CE de $5.11 \mathrm{dS} \mathrm{m}^{-1}$ ).

de nitrógeno y densidad de población se ajustaron a un modelo polinómico de segundo grado. La mayor producción de BT y RG por kg de nitrógeno aplicado y $5.11 \mathrm{dS} \mathrm{m}{ }^{-1}$, respectivamente. En los tres suelos, se observó que la fertilización de $100 \mathrm{~kg} \mathrm{ha}^{-1}$ de nitrógeno y densidad de 12 plantas $\mathrm{m}^{-2}$ promovió 

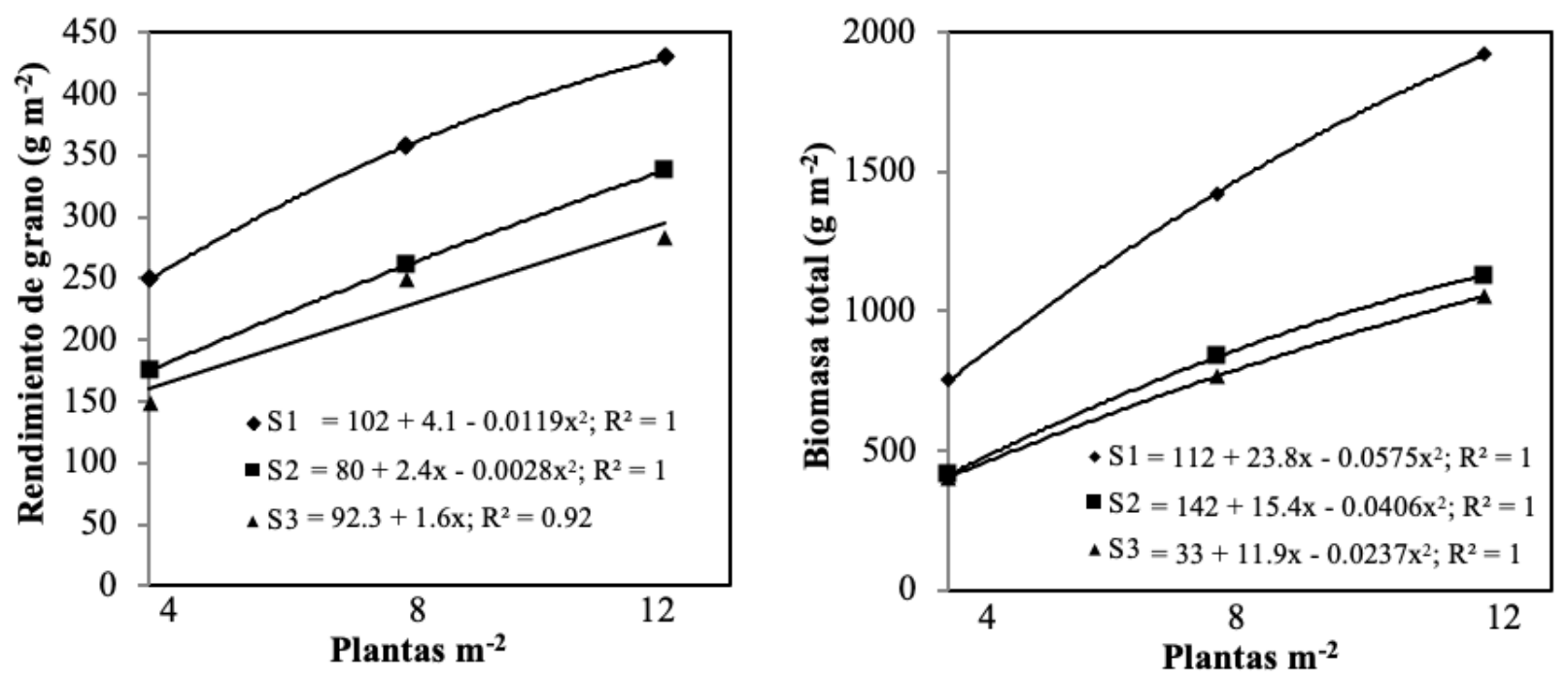

Figura 4. Biomasa total y rendimiento de grano del girasol en función a los niveles de nitrógeno y densidad de población DP4, DP8 y DP12 (4, 8 y 12 plantas m²).

la mayor producción de BT y RG, respecto a la nula fertilización y densidad de población 4 plantas $\mathrm{m}^{-2}$. Lo anterior indica que la fertilización nitrogenada es esencial para incrementar la BT y el RG, por ser el elemento más requerido por las plantas (Hawkesford et al. 2012; Morales et al. 2015); esto, a su vez, puede ser potencializado por un aumento en el número de plantas. Al respecto, Süzer (2010) observó que con la aplicación de $120 \mathrm{~kg} \mathrm{ha}^{-1}$ de nitrógeno y una densidad de 7 plantas $\mathrm{m}^{-2}$, incrementaron el RG en comparación con la cero fertilización y una densidad más alta (14 plantas $\mathrm{m}^{-2}$ ). Para el AC, el mayor tamaño se registró en la densidad más baja DP4, con $100 \mathrm{~kg}$ de $\mathrm{N} \mathrm{ha}^{-2}$ en los tres diferentes suelos; no obstante, de los tres suelos, el área del capítulo más extenso se presentó en $\mathrm{S} 1\left(452 \mathrm{~cm}^{2}\right)$. En cuanto a P100S, se encontró que en S1 el promedio del peso fue de $7.6 \mathrm{~g}$; en S2 fue de $6.8 \mathrm{~g}$ y en S3 de $5.6 \mathrm{~g}$, independientemente del nivel de nitrógeno y densidad de población, pues estadísticamente no se observaron diferentes entre ellos. Süzer (2010), al evaluar tres genotipos de girasol, tres niveles de nitrógeno $\left(0,60\right.$ y $\left.120 \mathrm{~kg} \mathrm{ha}^{-1}\right)$ y tres densidades de población $\left(7,9\right.$ y 14 plantas $\left.\mathrm{m}^{-2}\right)$ en un suelo ácido $(\mathrm{pH}$ 6.2), encontró que el mayor peso de 1,000 semillas fue con $0 \mathrm{~kg} \mathrm{~N}^{-1}$ y una densidad de 7 plantas $\mathrm{m}^{-2}$, contrario a lo observado en el presente estudio, donde el peso más alto se reportó con la aplicación de 50 y $100 \mathrm{~kg} \mathrm{ha}^{-1}$ de nitrógeno en las densidades de 8 y 12 plantas $\mathrm{m}^{-2}$, en el suelo con $\mathrm{pH}$ de 7.1.

\section{Conclusiones}

De acuerdo con los resultados de la presente investigación, la ocurrencia de las fases fenológicas no fue afectada por el tipo de suelo, fertilización nitrogenada y densidad de población. La producción de biomasa total y rendimiento de grano más alto se registró en el suelo (S1), con pH neutro y conductividad eléctrica de $0.49 \mathrm{dS} \mathrm{m}^{-1}$, fertilización nitrogenada de $100 \mathrm{~kg} \mathrm{ha}^{-1} \mathrm{y}$ una densidad de 12 plantas $\mathrm{m}^{-2}$. Para el área del capítulo, el mayor diámetro se registró con la aplicación $100 \mathrm{~kg} \mathrm{ha}^{-1}$ de nitrógeno y densidad de 4 plantas $\mathrm{m}^{-2}$ en el suelo 1 . En cuanto al P100S, los valores más altos se observaron en S1, así como para el índice de cosecha, donde la aplicación de $50 \mathrm{~kg} \mathrm{ha}^{-1}$ de nitrógeno y densidad de 4 plantas $\mathrm{m}^{-2}$ generó los mejores resultados. 
Cuadro 5. Biomasa total (BT), índice de cosecha (IC), rendimiento de grano (RG) y componentes del girasol Victoria en función al suelo, nitrógeno y densidad de población.

\begin{tabular}{|c|c|c|c|c|c|c|c|}
\hline SUELO & $\begin{array}{c}\mathrm{N} \\
\left(\mathrm{kg} \mathrm{ha}^{-1}\right)\end{array}$ & $\begin{array}{c}\mathrm{DP} \\
{\text { (plantas } \mathrm{m}^{-2} \text { ) }} \\
\end{array}$ & $\begin{array}{c}\mathrm{BT} \\
\left(\mathrm{g} \mathrm{m}^{-2}\right) \\
\end{array}$ & $\begin{array}{l}\text { IC } \\
(\%)\end{array}$ & $\begin{array}{c}\mathrm{RG} \\
\left(\mathrm{g} \mathrm{m}^{-2}\right)\end{array}$ & $\begin{array}{l}\text { P100S } \\
(\mathrm{g})\end{array}$ & $\begin{array}{c}\mathrm{AC} \\
\left(\mathrm{cm}^{2}\right)\end{array}$ \\
\hline \multirow{12}{*}{ S1 } & \multirow{3}{*}{0} & 4 & $637 \mathrm{i}^{\mathbb{I I}}$ & $22 \mathrm{c}$ & 226 e & $7 \mathrm{a}$ & $291 \mathrm{~b}$ \\
\hline & & 8 & $1,326 \mathrm{e}$ & $22 \mathrm{c}$ & $345 c$ & 7 a & $330 \mathrm{~b}$ \\
\hline & & 12 & $1,699 \mathrm{c}$ & $26 \mathrm{~b}$ & $425 \mathrm{a}$ & 8 a & $247 \mathrm{bc}$ \\
\hline & \multirow{3}{*}{50} & 4 & 713 hi & $35 \mathrm{a}$ & $271 \mathrm{~d}$ & 7 a & $338 \mathrm{~b}$ \\
\hline & & 8 & $1,412 \mathrm{e}$ & $24 \mathrm{bc}$ & $358 \mathrm{bc}$ & 8 a & $284 \mathrm{~b}$ \\
\hline & & 12 & $1,886 \mathrm{~b}$ & $22 \mathrm{c}$ & $424 \mathrm{a}$ & 8 a & $247 \mathrm{bc}$ \\
\hline & \multirow{3}{*}{100} & 4 & $896 \mathrm{~g}$ & $31 \mathrm{a}$ & 249 de & 8 a & $452 \mathrm{a}$ \\
\hline & & 8 & $1,542 \mathrm{~d}$ & $23 \mathrm{bc}$ & $371 \mathrm{bc}$ & $8 \mathrm{a}$ & $330 \mathrm{~b}$ \\
\hline & & 12 & $2,173 \mathrm{a}$ & $20 \mathrm{c}$ & 437 a & 8 a & $314 \mathrm{~b}$ \\
\hline & \multirow{3}{*}{0} & 4 & $325 \mathrm{k}$ & $31 \mathrm{a}$ & $150 \mathrm{~g}$ & $7 \mathrm{ab}$ & $213 c$ \\
\hline & & 8 & $803 \mathrm{gh}$ & $31 \mathrm{a}$ & $248 \mathrm{de}$ & $6 \mathrm{ab}$ & $182 \mathrm{c}$ \\
\hline & & 12 & $964 \mathrm{~g}$ & $25 \mathrm{bc}$ & $277 \mathrm{~d}$ & $6 \mathrm{~b}$ & $176 \mathrm{c}$ \\
\hline \multirow{6}{*}{ S2 } & \multirow{3}{*}{50} & 4 & $435 \mathrm{jk}$ & $31 \mathrm{a}$ & $185 \mathrm{f}$ & $7 \mathrm{ab}$ & $226 \mathrm{c}$ \\
\hline & & 8 & $824 \mathrm{gh}$ & $32 \mathrm{a}$ & $277 \mathrm{~d}$ & 8 a & $188 \mathrm{c}$ \\
\hline & & 12 & $1,112 \mathrm{f}$ & $32 \mathrm{a}$ & $355 \mathrm{~b}$ & $7 \mathrm{ab}$ & $164 \mathrm{~cd}$ \\
\hline & \multirow{3}{*}{100} & 4 & $476 j$ & $33 \mathrm{a}$ & $190 \mathrm{f}$ & 8 a & $240 \mathrm{c}$ \\
\hline & & 8 & $880 \mathrm{gh}$ & $31 \mathrm{a}$ & $257 \mathrm{~d}$ & $7 \mathrm{ab}$ & $219 c$ \\
\hline & & 12 & $1,239 \mathrm{e}$ & $29 \mathrm{~b}$ & $382 b c$ & $6 \mathrm{ab}$ & 195 c \\
\hline \multirow{11}{*}{ S3 } & \multirow{3}{*}{0} & 4 & $304 \mathrm{k}$ & $33 \mathrm{a}$ & $136 \mathrm{~g}$ & $6 \mathrm{ab}$ & $117 \mathrm{~d}$ \\
\hline & & 8 & 707 hi & $27 \mathrm{~b}$ & 209 ef & $6 \mathrm{ab}$ & $95 \mathrm{~d}$ \\
\hline & & 12 & $1,072 \mathrm{f}$ & $27 \mathrm{~b}$ & $276 \mathrm{~d}$ & $5 \mathrm{~b}$ & $67 \mathrm{~d}$ \\
\hline & \multirow{3}{*}{50} & 4 & $465 \mathrm{j}$ & $34 \mathrm{a}$ & $146 \mathrm{~g}$ & $6 \mathrm{ab}$ & $113 \mathrm{~d}$ \\
\hline & & 8 & $826 \mathrm{gh}$ & $31 \mathrm{a}$ & $264 d$ & $6 \mathrm{ab}$ & $74 \mathrm{~d}$ \\
\hline & & 12 & $1,155 \mathrm{f}$ & $24 \mathrm{bc}$ & $286 \mathrm{~d}$ & $6 \mathrm{~b}$ & $66 \mathrm{~d}$ \\
\hline & \multirow{3}{*}{100} & 4 & $469 j$ & $32 \mathrm{a}$ & $166 \mathrm{fg}$ & $6 \mathrm{ab}$ & $153 \mathrm{~d}$ \\
\hline & & 8 & $768 \mathrm{~h}$ & $32 \mathrm{a}$ & $273 d$ & $5 b$ & $95 \mathrm{~d}$ \\
\hline & & 12 & $1,232 \mathrm{e}$ & $24 \mathrm{bc}$ & $292 \mathrm{~d}$ & $6 \mathrm{~b}$ & $70 \mathrm{~d}$ \\
\hline & DMS & & 143.3 & 3.7 & 23.4 & 1.3 & 57.8 \\
\hline & $\mathrm{CV}$ & & 5.4 & 4.8 & 3.1 & 7.1 & 10.4 \\
\hline
\end{tabular}

"Letras distintas en columnas indican diferencias significativas, según Tukey = 0.05. P100S = Peso de 100 semillas; AC = Área del capítulo. Suelo 1 (S1), pH de 7.1 y CE de $0.49 \mathrm{dS} \mathrm{m}^{-1}$, suelo 2 (S2), pH de 7.8, CE de $1.75 \mathrm{dS} \mathrm{m}^{-1}$ y Suelo 3 (S3), pH de 8.1 y CE de $5.11 \mathrm{dS} \mathrm{m}^{-1}$. 


\section{Literatura Citada}

Ali A, Noorka IR. 2013. Nitrogen and phosphorus management strategy for better growth and yield of sunflower (Helianthus annuus L.) hybrid. Soil Environment 32: 44-48.

Allen GR, Pereira SL, Raes D, Smith M. 2006. Evapotranspiración del cultivo. Guia para la determinación de los requerimientos de agua de los cultivos. Organización de las Naciones Unidas para la Agricultura y la Alimentación. Roma.

Arenas-Julio YR, Escalante-Estrada JAS, Aguilar Carpio C, Rodríguez-González MT, Sosa-Montes E. 2021. Rentabilidad y rendimiento de girasol en función del tipo de suelo, nitrógeno y biofertilizante. Biotecnia 23: 45-51. https://doi.org/10.18633/biotecnia.v23i1.1284

Conus LA, Cardoso PC, Venturoso LR, Scalon SPQ. 2009. Germinação de sementes e vigor de plântulas de milho submetidas ao estresse salino induzido por diferentes sais. Revista Brasileira de Sementes 31: 67-74. https:// doi.org/10.1590/S0101-31222009000400008

Dos-Santos JB, Marenco-Centeno CR, Vieira de-Azevedo CA, Raj-Gheyi H, De-Lima GS, De-Lira VM. 2017. Crecimiento del girasol (Helianthus annuus L.) en función de la salinidad del agua de riego con fertilización nitrogenada. Agrociencia 51: 649-660.

Escalante-Estrada LE, Escalante-Estrada YI, LinzagaElizalde C. 2008. Densidad de siembra del girasol forrajero. Agronomía Costarricense 32: 177-182.

Escalante-Estrada JA, Rodríguez-González MT. 2010. Sunflower biomass distribution and seed yield in saline soil of Mexico highlands. HELIA 33: 127-134. https:// doi.org/10.2298/hel1052127e

Escalante-Estrada JAS, Rodríguez-González MT, EscalanteEstrada YI. 2015. Fenología, biomasa y rendimiento de cultivares de girasol en Valles Altos. Revista Mexicana de Ciencias Agrícolas 2: 307-311.

Escalante-Estrada JAS, Rodríguez-González MT, EscalanteEstrada YI. 2020. Distribución de biomasa y rendimiento en cultivares de girasol en función de la densidad de población y nitrógeno. Acta Agrícola y Pecuaria 6: E0061002. https://doi.org/10.30973/aap/2020.6.0061002

Flores MJ, Chan CJL, Bravo LA. 1985. Fenología del maíz y fríjol en el altiplano de Zacatecas II. Unidades calor (UC) y desarrollo fenológico. Fitotecnia 7: 66-81.

García E. 2004. Modificación al sistema de clasificación climática de Köppen. Universidad Nacional Autónoma de México. Distrito Federal, México.

García A, Quinche A. 2012. Girasol: respuesta a la aplicación foliar de boro en suelos del litoral suroeste de Uruguay. Agrociencia Uruguay 16: 7-14.

González LM, González MC, Ramírez R. 2002. Aspectos generales sobre la tolerancia a la salinidad en las plantas cultivadas. Cultivos Tropicales 23: 27-37.

Habeen N, Ahmad R. 2012. Improving tolerance of sunflower and safflower during growth stages to salinity through foliar spray of nutrient solutions. Pakistan Journal of Botany 44: 563-572.

Hawkesford M, Horst W, Kichey T, Lambers H, Schjoerring J, Moller IS, White P. 2012. Functions of macronutrients. En: Marschner P, editor. Marschners's Mineral Nutrition of Higher Plants. Adelaide, Australia. Academic Press. P. 135-189.

Morales MEJ, Morales-Rosales EJ, Díaz-Lopez E, CruzLuna AJ, Medina-Arias N, Guerrero-De la Cruz M. 2015. Tasa de asimilación neta y rendimiento de girasol en función de urea y urea de liberación lenta. Agrociencia 49: 163-176.

Morales-Rosales EJ, Escalante-Estrada JAS, López-Sandoval JA. 2008. Crecimiento, índice de cosecha y rendimiento de frijol (Phaseolus vulgaris L.) en unicultivo y asociado con girasol (Helianthus annuus L.). Universidad y Ciencia 24: 1-10. https://doi.org/10.19136/era.a24n1.274

Munns R, Goya SS, Passioura J. 2005. Salinity Stress and its Mitigation. University of California. Davis, USA.

Rasool FU, Hassan B, Jahangir IA. 2013. Growth and yield of sunflower (Helianthus annus L.) as influenced by nitrogen, sulphur and farmyard manure under temperate conditions. SAARC Journal of Agriculture 11: 81-89. https://doi.org/10.3329/sja.v11i1.18386

Sadras VO, Hall AJ. 1988. Quantification of temperature, photoperiod and population effect on plant leaf area in sunflower crop. Field Crops Research 18: 185-196. https://doi.org/10.1016/0378-4290(88)90008-1

Schneiter A, Miller JF. 1981. Description of sunflower growth stages. Crop Science 21: 901-903. https://doi. org/10.2135/cropsci1981.0011183X002100060024x

[SIAP] Servicio de Información Agroalimentaria y Pesquera. [internet]. 2018. Cierre de la producción agrícola de cultivos cíclicos y perennes 2018. [cited 2018 dic 20]. Disponible en: www. siap.gob.mx.

Silva TGFS, Zolnier JAS, Grossi JA, Barbosa JG, Moura CRW, Muniz MA. 2009. Crescimento do girassol ornamental cultivado em ambiente protegido sob difer- 
entes níveis de condutividade elétrica de fertirrigação.

Revista Ceres 56: 602-610.

Süzer S. 2010. Effects of nitrogen and plant density on dwarf sunflower hybrids. HELIA 33: 207-214. https: / / doi.org/10.2298/HEL1053207S 